INTERNATIONAL JOURN AL OF RESEARCHES IN BIOSCIENCES, AGRICULTURE AND TECHNOLOGY (c) VISHWASHANTI MULTIPURPOSE SOCIETY (Global Peace Multipurpose Socie ty) R. No. MH-659/13 (N) www.vmsindia.org

\title{
PLANT LEAF EXTRACTS AGAINST BLUE MOLD OF AMLA CAUESD BY PENICILLIUM ISLANDICUM A STORAGE FRUIT ROT
}

\author{
B. N. Sonawane ${ }^{1}$ and Fatima Sumia ${ }^{2}$ \\ ${ }_{1}^{1}$ Shri Dnyaneshwar Mahavidyalaya, Newasa Dist-Ahmednagar (M.S.) India. \\ ${ }^{2}$ Rafiqu Zakeria Women's College, Aurangabad (M.S.) India. \\ sbn3310@gmail.com
}

\begin{abstract}
:
Penicillium islandicum to know the fungi toxic nature of their plant extracts. Though complete inhibition of the pathogen was not observed in any of plant extracts tested, but considerable amount of inhibition was noticed in some of them. Among the $10^{\text {th }}$ plant extracts tested against Penicillium islandicum Tinospora cordifolia $(77.41 \%)$ were significantly superior over all other plant extracts. Next best was Datura stramomum (75.00\%), Agalecus mamrus (70.64 \% Tribulus teristrie (60.30\%), Adathoda vasica showed less inhibitory effect (6.66\%).
\end{abstract}

\section{Introduction}

Amla (Embilica officinallis) is one of the most important fruit plant grown in India. It is good source of vitamin- C. In order to regulate the supply and to enable the farmer to get a remunerative price for the produce, long term storage of amla is a pre requisite. Losses of amla during storage are considerable mainly due to sprouting and contamination by microorganisms. Nearly $30 \%$ of the production is losses during post harvest handling and sprouting, out of which microbial spoilage alone contributes approximately $15-20 \%$ of the total loss (Pantasticonanb Bantista, 1976; Bhagchandani et al., 1980).

In India Rajam 1992 reported that among the post-harvest disease of amla, Bule mold rot caused by Penicillium islandicum was the predominant one. Penicillium islandicum, a soil saprophyte being ubiquitous in occurre nce attacks amla by producing various enzymes and toxins and establishes itse lf in fruit, tissues. Chemically it controlled (Dang and Gupta 1984), but Chemical control may cause the poisoning to the amla fruits hence present study, an attempt has been made to find out suitable plant extracts which were used to control Penicillium islandicum and which will be free from hazardous chemical residues.

\section{Materials and Methods}

Penicillium islandicum was isolated from contaminated amla fruit. It was purified by single spore isolation technique (Riker and Riker, 1936) and maintained on PDA slants. Antifungal activities of different plant extracts were studied in vitro by poison food technique (Nene2000) (Datura stramomum, Azadirecta indica, Adathoda vasica, Acharanth usaspera, Amaranthus veridae, Boravia diffusa, Agalecus mamrus, Tinospora cordifolia, Tribulus teristries). $20 \mathrm{gm}$ fresh leaves and $20 \mathrm{ml}$ of distilled water $(\mathrm{w} / \mathrm{v})$ was taken while extraction. The extracted material was the $\mathrm{n}$ filtered through muslin cloth. The volume of extracted sap was made up to 50 $\mathrm{ml}$ by distilled water. The medium was the $\mathrm{n}$ sterilized $15 \mathrm{lbs}$. 20 minutes. The sterilized medium $20 \mathrm{ml}$ was poured in three plates equally. The petiriplates containing le af extracts were inoculated with loop holder of 10 days old fungal culture and incubated for ten days. Petiriplates with PDA medium acted as control. The petiriplates were observed on third day for inhibition of growth of Penicillium islandicum.

Table 1-Effect of plant extracts on radial mycelia growth of Penicillium islandicum.

\begin{tabular}{|c|c|c|c|c|}
\hline $\begin{array}{l}\text { Sr. } \\
\text { No }\end{array}$ & Treatment & Family & $\begin{array}{l}\text { Avera } \\
\text { ge } \\
\text { colon } \\
\text { y dia. } \\
(\mathrm{mm})\end{array}$ & $\begin{array}{l}\text { Inhibit } \\
\text { ion \% }\end{array}$ \\
\hline 1. & $\begin{array}{l}\text { Datura } \\
\text { stramomum L. }\end{array}$ & Solanaceae & 24.00 & 75.00 \\
\hline 2. & $\begin{array}{l}\text { Melia } \\
\text { azedarach }\end{array}$ & Meliacae & 38.00 & 43.20 \\
\hline 3. & $\begin{array}{l}\text { Tinospora } \\
\text { cordifolia } \\
\text { Miers }\end{array}$ & $\begin{array}{l}\text { Meniseerma } \\
\text { ceae }\end{array}$ & 21.33 & 77.51 \\
\hline 4. & $\begin{array}{l}\text { Tribulus } \\
\text { teristries L. }\end{array}$ & $\begin{array}{l}\text { Zygophylla } \\
\text { ceae }\end{array}$ & 36.50 & 60.30 \\
\hline 5. & $\begin{array}{l}\text { Amaranthsviri } \\
\text { de }\end{array}$ & $\begin{array}{l}\text { Amarantha } \\
\text { ceae }\end{array}$ & 44.00 & 47.80 \\
\hline 6. & $\begin{array}{l}\text { Acharanthu } \\
\text { saspera }\end{array}$ & $\begin{array}{l}\text { Amarantha } \\
\text { ceae }\end{array}$ & 54.66 & 35.90 \\
\hline 7. & $\begin{array}{l}\text { Withania } \\
\text { somanifera L. }\end{array}$ & Solanaceae & 52.00 & 37.70 \\
\hline 8. & $\begin{array}{l}\text { Agalecus } \\
\text { mamrus }\end{array}$ & Rutaceae & 20.33 & 70.64 \\
\hline 9. & $\begin{array}{l}\text { Adathoda } \\
\text { vasica }\end{array}$ & $\begin{array}{l}\text { Acanthace } \\
\text { ae }\end{array}$ & 86.00 & 06.66 \\
\hline 10. & $\begin{array}{l}\text { Azadirecta } \\
\text { indica L. }\end{array}$ & Meliaceae & 47.66 & 45.90 \\
\hline 11. & $\begin{array}{l}\text { Tamarindus } \\
\text { indica } L .\end{array}$ & $\begin{array}{l}\text { Caesalpina } \\
\text { ceae }\end{array}$ & 35.90 & 28.50 \\
\hline
\end{tabular}


Graph 1-Effect of plant extracts on radial mycelia growth of Penicillium islandicum.

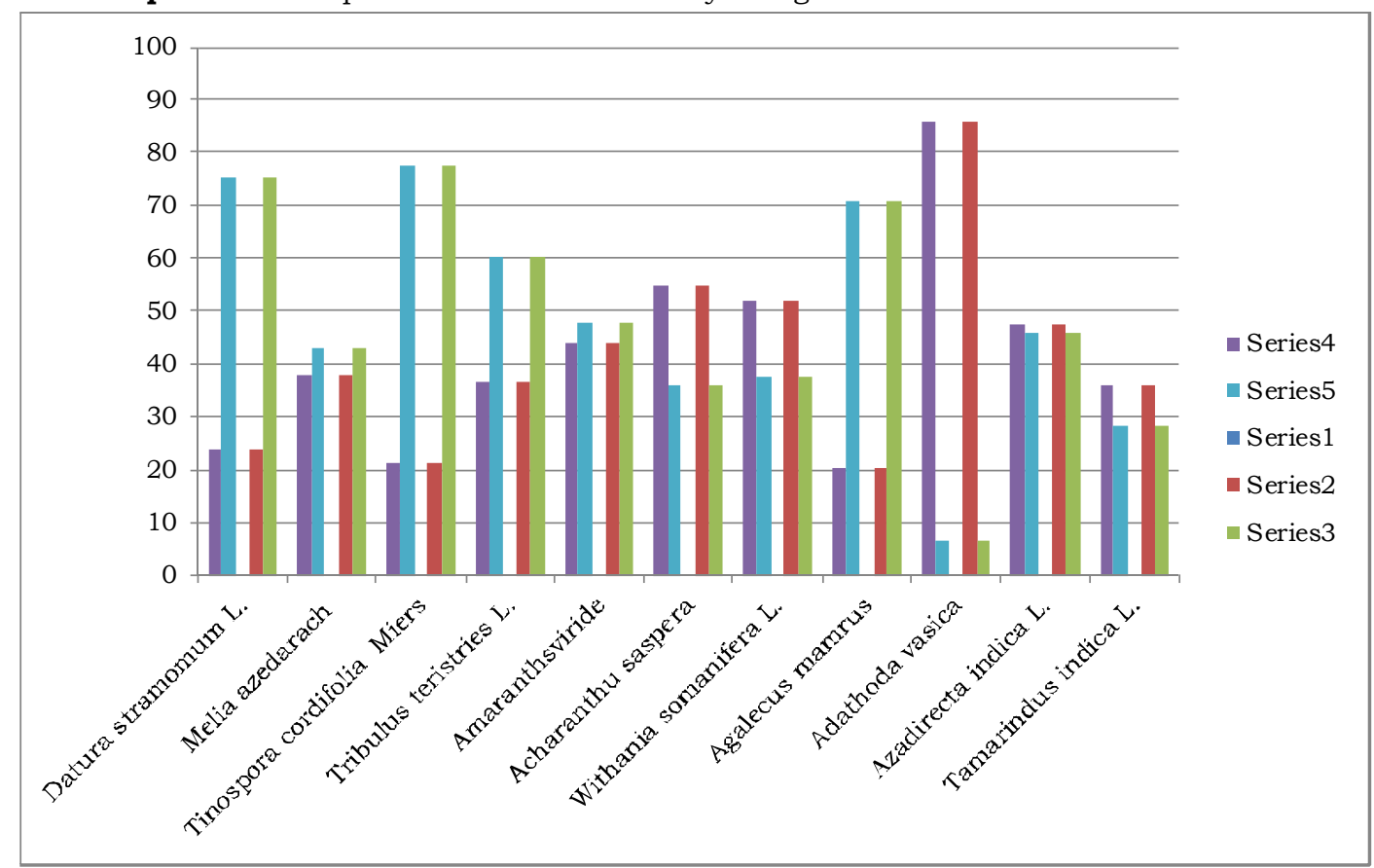

Table 2- Efficiency of leafextract of medicinal plants against the Penicillium islandicum.

\begin{tabular}{|l|c|c|c|c|c|c|}
\hline \multirow{2}{*}{ Plant extract } & \multicolumn{7}{|c|}{ Radial growth (mm) after days } \\
\cline { 2 - 7 } & $\mathbf{3}$ & $\mathbf{4}$ & $\mathbf{5}$ & $\mathbf{6}$ & $\mathbf{7}$ & Average \\
\hline Datura stramomum L. & 10 & 17 & 26 & 26.6 & 27 & 21.00 \\
\hline Melia azedarach & 8 & 10.3 & 13 & 21 & 23 & 15.6 \\
\hline Tinospora cordifolia & 4 & 8.3 & 12.6 & 19 & 20 & 12.78 \\
\hline Tribulus teristries & 7 & 15 & 19.6 & 26.6 & 36 & 20.84 \\
Amaranths viride & 6.3 & 12.6 & 38 & 40 & 46 & 28.58 \\
\hline Acharanthus aspera & 12 & 21.6 & 41 & 50 & 58 & 36.52 \\
\hline Withania somanifera L. & 15 & 24.6 & 41.6 & 48.6 & 56 & 37.36 \\
\hline Agalecus mamrus & 22 & 40 & 50 & 65.3 & 80 & 51.46 \\
\hline Adathoda vasica & 27 & 55.6 & 66.3 & 72.6 & 85 & 61.3 \\
\hline Azadirecta indica L. & 16 & 21.6 & 40.6 & 45.6 & 50 & 34.76 \\
\hline Tamarindus indica L & 18 & 22.10 & 40.15 & 48.20 & 50.15 & 35.72 \\
\hline Control & 28 & 56.3 & 72.3 & 80.6 & 90 & 65.44 \\
\hline
\end{tabular}

Graph 2- Efficiency of leafextract of me dicinal plants against the Penicilium islandicum.

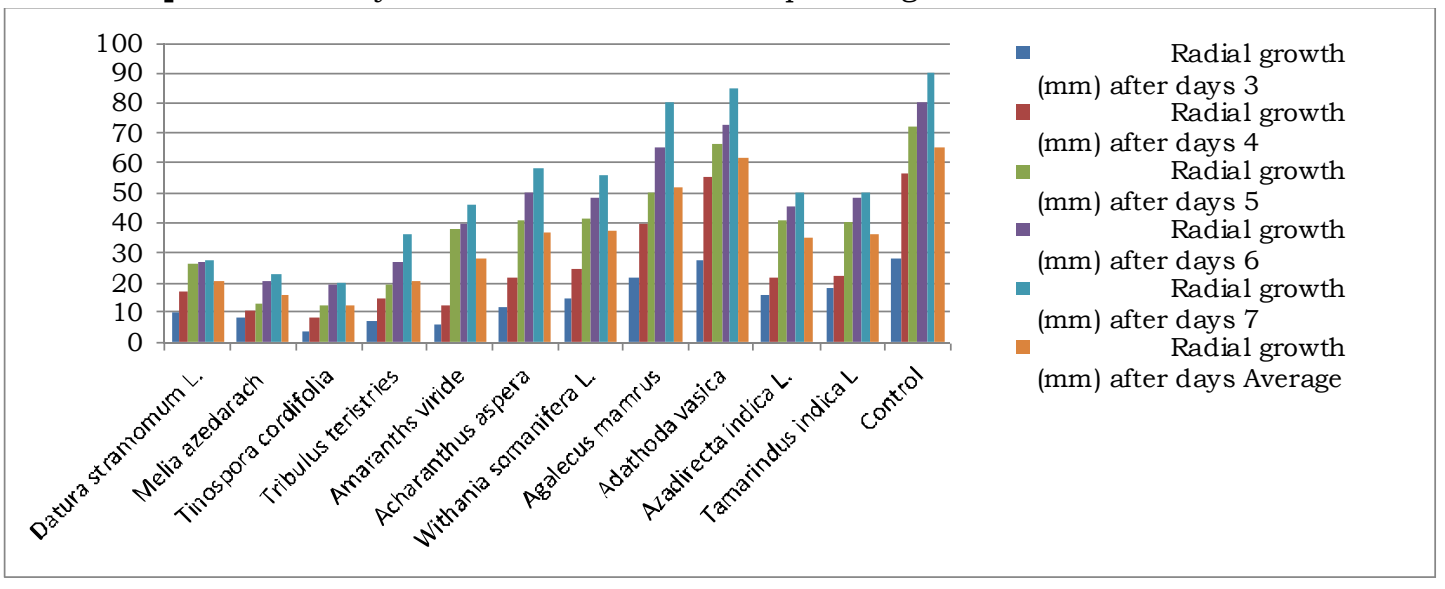




\section{Result and Discussion}

In present investigation plant extracts we re evaluate under in vitro condition against Penicillium islandicum to know the fungi toxic nature of theirplant extracts. Though complete inhibition of the pathogen was not observed in any of plant extracts tested, but conside rable amount of inhibition was noticed in some of them. Among the $10^{\text {th }}$ plant extracts tested against Penicillium islandicum Tinospora cordifolia $(77.51 \%)$ were significantly superior over all other plant extracts. Next best was Datura stramomum $L$. (75.00\%),Agalecus mamrus (70.64\%), Tribulus teristrie (60.30\%), Adathoda vasica showed less inhibitory effect $(6.66 \%)$.

\section{References}

1. Essono G, Ayodele M, Akoa A, Foko J, Olembo $\mathrm{S}$ and Gockwski J (2007). Aspergillus species on cassava chips in storage in rural areas of southern.Cameroon: their relationship with storage duration, moisture content. and processing methods African Joumal of Microbiology 00 1-008 (ㅁ)

2. VartikaRai and Shanta(2005). Toxic Contaminants in Herbal Drugs.Enviro. News 11 (吕)

3. Ananthanarayan and Paniker (1999). A Text Book of Practical Microbiology. 6th Edit (s)
4. Giridher P and Ready S M (1997). Incidences of mycotoxin producers on spices from Andhra Pradesh, J. Indian Bot. Soc. 76: 161-164 (ㅁ)

5. Bugno A, Adriana A B A, TatianaCP, TerezinhaAP, Myrna S (2006). Occurance of toxigenic fungi in herbal drugs.BrazillianJ rMicrobio 37: 47-51 (ㅁ)

6. Aziz NH, Youssef YA, Moheye Z EL-F, Lofty A M (1998). Contamination of some medicinal plant samples and spices by fungi and their mycotoxins.Bot. Bull Acad. Sin. 39: 279-2 85 (모)

7. Hitokoto H, Morozumi S, Wauke T, Saka, S, and Kurata $H$ (1978). Fungal contamination and mycotoxin detection of powdered herbal drugs. Applied and Environmental Microbiology 36: 252256 ( $\underline{s})$

8. Roy A K, Sinha KK and choursia H K(1988). Aflatoxin contamination of common medicinal plants. Applied and Environmental Microbiology 54: $842-843$ (ㅁ)

9. Efuntoye $\mathrm{MO}(2004)$. Fungi associated with herbal drug plants during storage. Mycopathologia 136: 115-118 (s)

10. Choursia H K(1990). Aflatoxin contamination in drug yielding plants. J. Indian Bot. Soc. 69: 281-283 (ㅁ)

11. Kumar S and Roy A K (1994). Mycotoxin contamination of some kidney curative herbal drugs. Indian Bot. Soc. 73: 341-342 (ㅁ). 